\title{
A Practical Method to Cleave Diphenyl Phosphonate Esters to Their Corresponding Phosphonic Acids in One Step
}

\author{
Bo-Seung Kim, ${ }^{\dagger}$ Beom-Tae Kim, ${ }^{\ddagger}$ and Ki-Jun Hwang ${ }^{\star}$ \\ Department of Chemistry, Chonbuk National Lniversity, Chonju 561-756, Korea \\ ${ }^{\dagger}$ Department of Bioactive Material Sciences, College of Natural Science, Chonbuk National Cniversity. \\ Chonit 561-756, Korea \\ ${ }^{\ddagger}$ Research Center of Bioactive Materials, Chonbuk National Lniversity. Chonju 561-756, Korea \\ 'E-mail kitunachonbuk.ackr \\ Received March 13, 2009, Accepted April 22, 2009
}

Key Words: Diphenylphosphonate esters, Diphenylphosphonic acids, Hydrolysis, Anmonium fluoride

During a study conducted to evaluate the synthesis of nucleoside phosphonic acids as potential antiviral agents ${ }^{\mathrm{l}}$ and/ or antitumor agents. ${ }^{2}$ it was necessary to hydrolyze the diphenyrlphosphonate ester 1 to its phosphonic acid 2 under mild conditions while maintaining a good yield (Scheme 1). To our surprise. there were no previous reports describing the direct cleavage of diphenylphosphonate esters of nucleosides into their corresponding phosphonic acids under mild reaction conditions in one step. Indeed, a thorough literature study revealed quite a few limited methods that employed indirect and multistep processes that are shown in Scheme 2. These examples clearly demonstrated that a direct procedure to hydrolyze the diphenyl phosphonate esters to their phosphonic acids in one step is not straightforward. The other previously described methods utilized either very harsh reaction conditions ( $30 \% \mathrm{HCl}$ reflux. $2 \mathrm{~h}$ ). or hydrogenolysis using Adams' catalyst $\left(\mathrm{H}_{2} / \mathrm{PtO}_{2}\right){ }^{6}$ which was not recommended even by the authors of the study that described the method. Based on the aforementioned background. a mild and direct one step liydrolysis (cleavage) of diphenylphosphonate esters to their corresponding phosphonic acids is warranted.

After several unsuccessful attempts, we found that nucleoside 1 can be converted to nucleoside 2 in $>80 \%$ yield simply by treating 1 with 10 eq. of ammonium fluoride in $\mathrm{CH}_{3} \mathrm{CN} / \mathrm{H}_{2} \mathrm{O}$ $(1: 1, v / v)$ at $60^{\circ} \mathrm{C}$ for 2 hours. All other simple attempts to utilize either acid or base hydrolysis were unsuccessful. Encouraged by our findings, to explore a generality of this result and extend the scope of this useful reaction several other diphenyl phosphonate esters $\mathbf{5}$ as simple substrates were prepared by treating aldehydes 3 with dipheny l triphenylphosphoranylidenemethylphosphonate 4 . which resulted in a yield of $>85 \%$ (Scheme 3). It is important to note that the phosphonium y lide + stabilized by a diarylphosphonate group provided exclusively trans isomers $\mathbf{5}$. The diphenylphosphonate esters 5 were then subjected to our reaction conditions $\left(\mathrm{NH}_{4} \mathrm{~F}, \mathrm{CH}_{2} \mathrm{CN} / \mathrm{H}_{2} \mathrm{O}(\mathrm{l}: \mathrm{l}, \mathrm{v} / \mathrm{v}) .60^{\circ} \mathrm{C} .2 \mathrm{~h}\right)$ to provide the expected free phosphonic acids 6 with good yields (Table 1). The<smiles>C=CC(=O)C1OC(CO)C(O)C1n1cnc2c(N)ncnc21</smiles>

Adenosine<smiles>[R]Nc1ncnc2c1ncn2C1OC([R4])C(C=CP(=O)(Oc2ccccc2)Oc2ccccc2)O1</smiles>

$1 \mathrm{R}^{1}=\mathrm{H}, \mathrm{R}^{2}=\mathrm{OH}$<smiles>[R1]Nc1ncnc2c1ncn2[C@@H]1O[C@@H](/C=C/P(=O)(O)O)[C@@H]([R2])[C@@H]1N</smiles>

$2 \mathrm{R}^{1}=\mathrm{H}_{1} \mathrm{R}^{2}=\mathrm{OH}$

Scheme 1

$1)^{3}$<smiles>[R]CP(=O)(Oc1ccccc1)c1ccccc1</smiles>

$2)^{4}$<smiles>[R]CP(=O)(O)c1ccccc1</smiles>
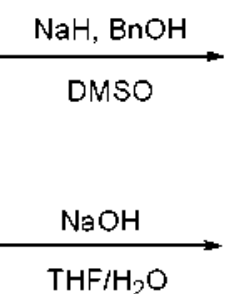<smiles>[R]CP(=O)(O)Oc1ccccc1</smiles>

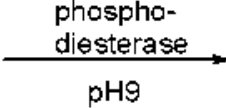<smiles>[R]CP(=O)(O)O</smiles>

$R, R^{\prime}=$ nucleosides 


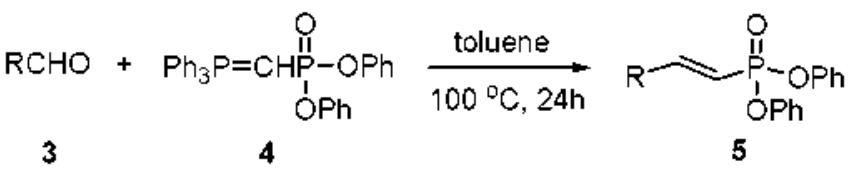

Scheme 3

Table 1. Hydrolysis of diphenylphosphonate esters 5 to phosphonic acids 6 .

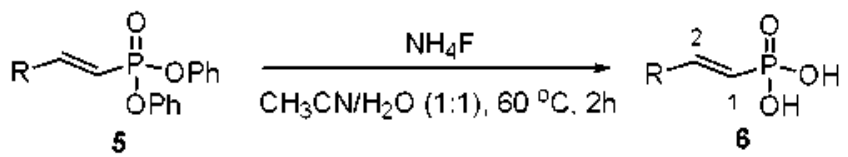

\begin{tabular}{cclccc}
\hline Entry & Substrate & Substituent (R) & Product & $\begin{array}{c}\text { Reaction } \\
\text { Time (h) }\end{array}$ & $\begin{array}{c}\text { Yield } \\
(\%)\end{array}$ \\
\hline 1 & $\mathbf{5 a}$ & phenyl & $\mathbf{6 a}$ & 3 & 85 \\
2 & $\mathbf{5 b}$ & p-chlorophenyl & $\mathbf{6 b}$ & 2 & 90 \\
3 & $\mathbf{5 c}$ & p-nitrophenyl & $\mathbf{6 c}$ & 2 & 95 \\
4 & $\mathbf{5 d}$ & p-tolyl & $\mathbf{6 d}$ & 2 & 95 \\
5 & $\mathbf{5 e}$ & benzyl & $\mathbf{6 e}$ & 3 & 80 \\
6 & $\mathbf{5 f}$ & n-butyl & $\mathbf{6 f}$ & 3 & 83 \\
7 & $\mathbf{5 g}$ & iso-butyl & $\mathbf{6 g}$ & 2 & 85 \\
8 & $\mathbf{1}$ & adenosyl & $\mathbf{2}$ & 2 & 80 \\
\hline
\end{tabular}

structure of compounds 6 and 2 were fully confirmed by instrumental analy sis of the ${ }^{1} \mathrm{H},{ }^{13} \mathrm{C}-\mathrm{NMR}$ and high resolution mass spectra. The results shown in Table I deserve some mention. Thus, the substrates of compound $\mathbf{5}$, which were designed carefully so that they contained electron withdrawing (5c). electron donating (5d), benzylic (5e), simple aliphatic (5f) and sterically hindered aliphatic groups $(\mathbf{5 g})$. did not affect the yields and the reaction time under the given conditions.

In summary. this mild and convenient reaction condition hydrolyzed the diphenyl phosphonate esters to their corresponding free phosphonic acids with excellent results. Accordingly this method could be utilized for the preparation of quite complicated compounds containing phosphonic acid groups. such as compound 2.

\section{Experimental Section}

Melting points were recorded on Electrothermal melting point apparatus and were uncorrected. Mass spectra were recorded on Sy'napt HDMS (Waters). ${ }^{1} \mathrm{H}$ - and ${ }^{13} \mathrm{C}-\mathrm{NMR}$ spectra were recorded on Jeol $400 \mathrm{MHz}$ or $600 \mathrm{MHz}$ spectrometer. Chemical shifts are shown in $\hat{o}$ values (ppm) with tetramethylsilane (TMS) as internal standard

Genemal procedure for the hydrolysis. A solution of compound 5 a (50 $\mathrm{mg} .0 .15 \mathrm{mmol}$ ) and $\mathrm{NH}_{4} \mathrm{~F}$ (55 $\mathrm{mg}, 1.5 \mathrm{mmol}$ ) in $2 \mathrm{~mL}$ of $\mathrm{CH}_{3} \mathrm{CN} / \mathrm{H}_{2} \mathrm{O}(1: 1, v / \mathrm{v})$ was stirred at $60^{\circ} \mathrm{C}$. After 3 hours. the reaction mixture was concentrated. loaded onto an anionic DOWEX $1 \times 4-200$ column. washed with three volumes of $\mathrm{H}_{2} \mathrm{O}$ and then eluted with $0.1 \mathrm{Naq}$. HCl. The collected fractions determined to contain product by TLC analysis were then subjected to freeze drying to produce compound $6 \mathbf{a}$ as a white solid ( $23 \mathrm{mg} .85 \%$ yield): $\mathrm{mp}>320^{\circ} \mathrm{C}$ (dec.): ${ }^{1} \mathrm{H}$ NMR $\left(400 \mathrm{MHz}, \mathrm{D}_{2} \mathrm{O}\right) \hat{o} 6.33\left(\mathrm{dd}, 1 \mathrm{H}, J_{\mathrm{H} . \mathrm{H}}=17.6 \mathrm{~Hz} . J_{\mathrm{P} . \mathrm{H} . \mathrm{gent}}=15.6\right.$ $\mathrm{Hz} . \mathrm{H}-\mathrm{l}$ ). 6.99 (dd. IH. $J_{\mathrm{P}, \mathrm{H}, \mathrm{vic}}=20.4 \mathrm{~Hz}, J_{\mathrm{H} . \mathrm{H}}=17.6 \mathrm{~Hz}, \mathrm{H}-2$ ). 7.42-7.20 (m. 5H. H-Ar): ${ }^{13} \mathrm{C} \mathrm{NMR}\left(100 \mathrm{MHz}, \mathrm{D}_{2} \mathrm{O}\right) \delta 129.80$. $130.53(\mathrm{~m}) .130 .82 .131 .61,131.79 .143 .72(\mathrm{n})$ ) HRMS (ESI): $m z \mathrm{z} 185.0370[\mathrm{M}+\mathrm{H}]^{-} . \mathrm{C}_{8} \mathrm{H}_{1} \mathrm{O}_{2} \mathrm{P}$ requires 185.0368: Compound 6b mp > $320^{\circ} \mathrm{C}$ (dec). ${ }^{1} \mathrm{H}$ NMR $\left(400 \mathrm{MHz}, \mathrm{D}_{2} \mathrm{O}\right) \delta 6.32$ (dd,

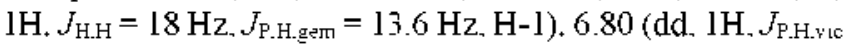
$\left.=18 \mathrm{~Hz}, J_{\mathrm{H}, \mathrm{H}}=18 \mathrm{~Hz}, \mathrm{H}-2\right), 7.22$ (d. $2 \mathrm{H}, J=8.8 \mathrm{~Hz}, \mathrm{H}-\mathrm{Ar}$ ), 7.34 (d. $2 \mathrm{H}, J=8.8 \mathrm{~Hz}$. H-Ar): ${ }^{13} \mathrm{C} \mathrm{NMR}\left(100 \mathrm{MHz}, \mathrm{D}_{2} \mathrm{O}\right) \delta$ 129.87, 130.29. 130.77, 130.95. 134.23 (m). 138.47 (m): HRMS (ESI): $m z 218.9981[\mathrm{M}+\mathrm{H}]^{+} . \mathrm{C}_{8} \mathrm{H}_{9} \mathrm{ClO}_{3} \mathrm{P}$ requires 218.9978 ; Compound 6e mp 129-130 ${ }^{\circ} \mathrm{C}:{ }^{1} \mathrm{H} N \mathrm{NMR}\left(400 \mathrm{MHz}, \mathrm{D}_{2} \mathrm{O}\right) \hat{\delta}$ 6.47 (dd. $\left.1 \mathrm{H}, J_{\mathrm{H} . \mathrm{H}}=17.6 \mathrm{~Hz}, J_{\mathrm{F} . \mathrm{H} . \mathrm{gen}_{\mathrm{H}}}=16.4 \mathrm{~Hz}, \mathrm{H}-1\right) .7 .21(\mathrm{dd}$, lH. $\left.J_{\mathrm{P} . \mathrm{H} . \text { ic }}=22.4 \mathrm{~Hz}, J_{\mathrm{H} . \mathrm{H}}=17.6 \mathrm{~Hz}, \mathrm{H}-2\right) .7 .54$ (d. $2 \mathrm{H} . J=8.4$ Hz. H-Ar). 8.03 (d. $2 \mathrm{H} . J=8.4 \mathrm{~Hz} . \mathrm{H}-\mathrm{Ar}$ ): ${ }^{13} \mathrm{C}$ NMR (100 $\left.\mathrm{MHz}, \mathrm{D}_{2} \mathrm{O}\right)$ o o $123.72(\mathrm{~m}) .126 .68,130.95 .132 .50(\mathrm{~m}), 145.65$ (m), 150.43: HRMS (ESI): $m z 230.0221[\mathrm{M}+\mathrm{H}]^{-}, \mathrm{C}_{8} \mathrm{H}_{9} \mathrm{NO}_{5} \mathrm{P}$ requires 230.0218; Compound $6 \mathrm{~d} \mathrm{mp} 212-213{ }^{\circ} \mathrm{C}$ : ${ }^{1} \mathrm{H}$ NMR $\left(400 \mathrm{MHz}, \mathrm{D}_{2} \mathrm{O}\right) \delta \delta 2.21$ (s. $\left.3 \mathrm{H}\right), 6.27$ (dd. $1 \mathrm{H}, J_{\mathrm{H}, \mathrm{H}}=18 \mathrm{~Hz}$. $\left.J_{\text {P.H.gem }}=18 \mathrm{~Hz} \cdot \mathrm{H}-1\right) .7 .14$ (d, $\left.2 \mathrm{H} . J=8 \mathrm{~Hz} . \mathrm{H} \cdot \mathrm{Ar}\right) .7 .19(\mathrm{dd}$, $\left.1 \mathrm{H} . J_{\mathrm{F} \cdot \mathrm{H} v \mathrm{ic}}=23.2 \mathrm{~Hz} . J_{\mathrm{HH}}=18 \mathrm{~Hz}, \mathrm{H}-2\right), 7.36(\mathrm{~d}, 2 \mathrm{H} . J=8 \mathrm{~Hz}$, H-Ar): ${ }^{13} \mathrm{C}$ NMR (100 MHz, D 20$) \delta 117.67$ (m). 130.25. 132.29. $135.18(\mathrm{~m}), 1+3.52 .1+8.45(\mathrm{~m})$ : HRMS (ESI): $m z$ $199.0529[\mathrm{M}+\mathrm{H}]^{-}, \mathrm{C}_{9} \mathrm{H}_{12} \mathrm{O}_{2} \mathrm{P}$ requires 199.0524: Compound 6e mp 145-146 ${ }^{\circ} \mathrm{C}$ : ${ }^{1} \mathrm{H}$ NMR $\left(400 \mathrm{MHz}, \mathrm{D}_{2} \mathrm{O}\right)$ ô 2.60 (dd, $1 \mathrm{H}$, $J=7.2 \mathrm{~Hz}, 4 \mathrm{~Hz}, \mathrm{H}-3 \mathrm{a}$ ), 2.66 (dd. $\mathrm{lH}, J=7.2,+4 \mathrm{~Hz}, \mathrm{H}-3 \mathrm{~b}$ ). 6.14 (m. IH. H-1), 6.47 (m, IH. H-2). 7.15-7.35 (m, 5H, H-Ar): ${ }^{12} \mathrm{C}$ NMR (100 MHz. D_O) ô $33.23(\mathrm{~m}) .123 .66(\mathrm{~m}) .128 .83$ (m). 130.34. $131.58(\mathrm{~m}) .136 .15(\mathrm{~m}), 139.83$ (m): HRMS (ESI): $m z 199.0531[\mathrm{M}+\mathrm{H}]^{-}, \mathrm{C}_{9} \mathrm{H}_{1} \mathrm{O}_{3} \mathrm{P}$ requires 199.0524; Compound $\left.6 \mathrm{f}^{\mathrm{m}} \mathrm{mp}>180^{\circ} \mathrm{C}(\mathrm{dec})\right)^{1} \mathrm{H}$ NMR $\left(400 \mathrm{MHz}, \mathrm{D}_{2} \mathrm{O}\right) \delta 80.76$ (t, $3 \mathrm{H}, J=13.2 \mathrm{~Hz}, \mathrm{H}-6$ ). 1.20 (dd. $2 \mathrm{H}, J=13.2 \mathrm{~Hz}, 6 \mathrm{~Hz}$, $\mathrm{H}-5$ ). 1.28 (dd. $2 \mathrm{H} . J=13.2 \mathrm{~Hz} .6 \mathrm{~Hz} . \mathrm{H}-4) .2 .26(\mathrm{~d}, 1 \mathrm{H}, J=$ $6 \mathrm{~Hz}, \mathrm{H}-3), 5.55$ (dd. $1 \mathrm{H} . J_{\mathrm{P}, \mathrm{H}, \mathrm{gem}}=18.8 \mathrm{~Hz}, J_{\mathrm{H}, \mathrm{H}}=13.2 \mathrm{~Hz}$. $\mathrm{H}-1$ ). 6.29 (ddd. $1 \mathrm{H} . J_{\mathrm{P} . \mathrm{H} . v_{\mathrm{ic}}}=52.8 \mathrm{~Hz}, J_{\mathrm{H} . \mathrm{H}}=13.2 \mathrm{~Hz}, 6 \mathrm{~Hz}$, $\mathrm{H}-2):{ }^{13} \mathrm{C}$ NMR (100 MHz, D 0 ) o $23.57,29.10,44.88$ (m). $121.04(\mathrm{~m}) .152 .77(\mathrm{~m})$ ) HRMS (ESI): $m z 165.0685[\mathrm{M}+\mathrm{H}]^{+}$, $\mathrm{C}_{6} \mathrm{H}_{14} \mathrm{O}_{5} \mathrm{P}$ requires 165.0681: Compound $6 \mathrm{~g}$ mp 88-89 ${ }^{\circ} \mathrm{C}:{ }^{3} \mathrm{H}$ NMR (400 MHz, D2O) $\delta 0.76$ (s. 3H. H-methyl), 0.77 (s. $3 \mathrm{H}$. $\mathrm{H}$-methyl), $1.62(\mathrm{sp}, 1 \mathrm{H}, J=7.6 \mathrm{~Hz} . \mathrm{H}-4) .1 .98$ (t. $2 \mathrm{H}, J=7.6$ Hz. H-3). 5.63 (dd. IH. $J_{\mathrm{P}, \mathrm{H} \text {,gem }}=21.6 \mathrm{~Hz}, J_{\mathrm{H}, \mathrm{H}}=17.2 \mathrm{~Hz}$. $\mathrm{H}-1$ ). 6.47 (ddd. $1 \mathrm{H}, J_{\mathrm{P} . \mathrm{H}, v_{\mathrm{ic}}}=30 \mathrm{~Hz}, J_{\mathrm{H} . \mathrm{H}}=17.2 \mathrm{~Hz}, 7.6 \mathrm{~Hz}$. $\mathrm{H}-2):{ }^{13} \mathrm{C} \mathrm{NMR}\left(100 \mathrm{MHz}, \mathrm{D}_{2} \mathrm{O}\right)$ ô $15.23,23.74,32.51 .32 .53$. $119.52(\mathrm{nl}) .153 .59(\mathrm{~nm})$; HRMS (ESI): $m z 165.0673[\mathrm{M}+\mathrm{H}]^{+}$, $\mathrm{C}_{6} \mathrm{H}_{14} \mathrm{O}_{5} \mathrm{P}$ requires 165.0681 .

Preparation of compound 2. Compound 1 (28 mg. 0.54 minol) was treated by the same procedure used to prepare compound 6: however. the anionic DOWEX $1 \times 4-200$ column was substituted with DEAE Sephadex (eluent $50 \mathrm{mM}$ anmonium bicarbonate). This procedure yielded compound $\mathbf{2}$ as a white solid (17 mg. 80\% yield): ${ }^{1} \mathrm{H}$ NMR $\left(400 \mathrm{MHz}, \mathrm{D}_{2} \mathrm{O}\right) \delta$ $4.24(\mathrm{t}, 1 \mathrm{H} . J=4.8 \mathrm{~Hz}), 4.50(\mathrm{~s}, 1 \mathrm{H}) .4 .86(\mathrm{t}, 1 \mathrm{H}, J=4.8 \mathrm{~Hz})$, $5.88($ d. $1 \mathrm{H} . J=4.8 \mathrm{~Hz}) .5 .94$ (d. IH. $\left.J_{\mathrm{H} . \mathrm{H}}=17.2 \mathrm{~Hz} . \mathrm{H}-\mathrm{l}\right)$. 6.52 (ddd. $1 \mathrm{H} . J_{\text {P.H. } . \mathrm{em}}=21.6 \mathrm{~Hz}, J_{\mathrm{H} . \mathrm{H}}=17.2 \mathrm{~Hz} .4 .8 \mathrm{~Hz} . \mathrm{H}-2$ ), 7.99 (s. lH), 8.06 (s. IH): ${ }^{13} \mathrm{C} \mathrm{NMR}\left(100 \mathrm{MHz} . \mathrm{D}_{2} \mathrm{O}\right) \delta 66.63$. $75.47,83.87(\mathrm{~m}), 90.00,124.19(\mathrm{~m}), 131.74,142.32,145.84$ (m). 150.94, 154.06, 156.97; HRMS (ESI): $m z 371.0997$ $[\mathrm{M}+3 \mathrm{H}]^{+} . \mathrm{C}_{6} \mathrm{H}_{14} \mathrm{O}_{3} \mathrm{P}$ requires 371.0981 
Acknowledgments. This work was supported by the grant from Duk-myung. Huh Jin-Kyu Memorial Fund of Chonbuk National University. The authors thank Dr. Joseph Kwon at Korea Basic Science Institute, Kwangju Branch for providing the high resolution mass spectra.

\section{References}

1. Boojamra, C. G.: Mackman, R. L.: Markevitch, D. Y.: Prasad, V:; Ray, A. S.: Douglas, T.; Grant, D.; Kim C. U.: Cihlar, T. Bioorg. Med. Chem. Lett. 2008, 18, 1120

2. (a)Leblond, L.: Attardo, G.: Hamelin, B.: Bouffard, D. Y.:
Nouyen-Ba, N.: Gourdean, H. Mol. Cancer Ther. 2002, 1, 737. (b) Hajek, M.; Matulova, N.; Votruba, I.; Holy, A.; Tloust'ova, E. Biochem. Phamacol 2005, 70, 894.

3. Koh, Y. H.: Shim, J. H.: Wul, I. Z:; Zhong, W.; Hong, Z.; Girardet, T. L. I. Med Chem 2005, $48,2867$.

4. Freeman, G. A.; Rideout, J. L.; Miller, W. H.; Reardon, J. E. $J$. Med. Chem. 1992. 35. 3192

5. Fanq. A. H.; Khan, M. A.; Eckman, C. Synth. Commun. 2004. 34.775

6. Lejczak, B.; Kafarski, P. Symthesis 1982, 412.

7. Iones, G. H.: Hamamma, E. K.: Moffatt, I. G. Tetrahedron Lett. $1968,55,5731$. 